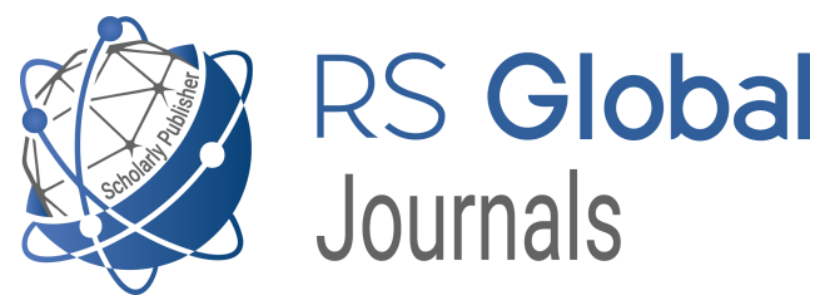

Scholarly Publisher RS Global Sp. z O.O. ISNI: 0000000484952390

Dolna 17, Warsaw, Poland 00-773

Tel: +48226022703

Email: editorial_office@rsglobal.pl

\begin{tabular}{ll} 
JOURNAL & Science Review \\
\hline p-ISSN & $2544-9346$ \\
\hline e-ISSN & $2544-9443$ \\
\hline PUBLISHER & RS Global Sp. z O.O., Poland
\end{tabular}

ARTICLE TITLE

$\operatorname{AUTHOR}(\mathbf{S})$

ARTICLE INFO

DOI

RECEIVED

ACCEPTED

PUBLISHED

LICENSE
INTERVAL EDGE COLORING OF TREES WITH STRICT RESTRICTIONS ON THE SPECTRUMS

Albert Khachik Sahakyan

Albert Khachik Sahakyan. (2021) Interval Edge Coloring of

Trees with Strict Restrictions on the Spectrums. Science Review. 3(38). doi: 10.31435/rsglobal_sr/30072021/7592

https://doi.org/10.31435/rsglobal_sr/30072021/7592

19 April 2021

10 June 2021

15 June 2021

\section{(c) (i)}

This work is licensed under a Creative Commons Attribution

\subsection{International License.}

(C) The author(s) 2021. This publication is an open access article. 


\title{
INTERVAL EDGE COLORING OF TREES WITH STRICT RESTRICTIONS ON THE SPECTRUMS
}

\author{
Albert Khachik Sahakyan, Chair of Discrete Mathematics and Theoretical Informatics, Faculty of \\ Informatics and Applied Mathematics, Yerevan State University, Armenia
}

DOI: https://doi.org/10.31435/rsglobal_sr/30072021/7592

\section{ARTICLE INFO}

Received 19 April 2021

Accepted 10 June 2021

Published 15 June 2021

\section{KEYWORDS}

trees, interval t-coloring, interval edge coloring, restrictions on spectrums, dynamic programming.

\begin{abstract}
An edge-coloring of a graph $G$ with consecutive integers $c_{1}, \ldots, c_{t}$ is called an interval t-coloring if all the colors are used, and the colors of edges incident to any vertex of $G$ are distinct and form an interval of integers. A graph $G$ is interval colorable if it has an interval t-coloring for some positive integer $t$. For an edge coloring $\alpha$ and a vertex $v$ the set of all the colors of the incident edges of $v$ is called the spectrum of that vertex in $\alpha$ and is denoted by $S_{\alpha}(v)$. We consider the case where the spectrum for each vertex $v$ is provided $S(v)$, and the problem is to find an edge-coloring $\alpha$ such that for every vertex $v, S_{\alpha}(v)=S(V)$. We provide an $O(N)$ algorithm that finds such an edge-coloring for trees that satisfies all the restrictions. If it is impossible to have an edge-coloring that satisfies the restrictions of the spectrums the algorithm will tell that too.
\end{abstract}

Citation: Albert Khachik Sahakyan. (2021) Interval Edge Coloring of Trees with Strict Restrictions on the Spectrums. Science Review.3(38). doi: 10.31435/rsglobal_sr/30072021/7592

Copyright: (C) 2021 Albert Khachik Sahakyan. This is an open-access article distributed under the terms of the Creative Commons Attribution License (CC BY). The use, distribution or reproduction in other forums is permitted, provided the original author(s) or licensor are credited and that the original publication in this journal is cited, in accordance with accepted academic practice. No use, distribution or reproduction is permitted which does not comply with these terms.

Introduction. All graphs considered in this paper are undirected (unless explicitly said), finite, and have no loops or multiple edges. For an undirected graph $G$, let $V(G)$ and $E(G)$ denote the sets of vertices and edges of $G$, respectively. The degree of a vertex $v \in V(G)$ is denoted by $d_{G}(v)$, the maximum degree of vertices by $\Delta(G)$. Let $T$ be a tree (a connected undirected acyclic graph).

For a directed graph $\vec{G}$ if there is an edge from a vertex $u$ to a vertex $v$ we will denote it as $u \rightarrow v$ or $(u, v) \in E(\vec{G})$. The graph $G$ is called the underlying undirected graph of a directed graph $\vec{G}$ if $V(G)=$ $V(\vec{G})$ and $E(G)=\{(u, v) \mid$ iff $u \rightarrow v$ or $v \rightarrow u\}$ (between any pair of vertices $u$ and $v$, if the directed graph has an edge $u \rightarrow v$ or an edge $v \rightarrow u$, the underlying undirected graph includes the edge $(u, v)$ ).

For a tree $T$ and a vertex $r$ let $T_{r}$ be the directed graph whose underlying undirected graph is $T$ and in $T_{r}$ each edge is directed in such a way that for all vertices $v \in T_{r}$ there is a path in $T_{r}$ from $r$ to $v$. We will say that $T_{r}$ is a rooted tree with the root $r$. Fig. 1 illustrates the rooted tree $T_{v_{1}}$ with the root $v_{1}$.

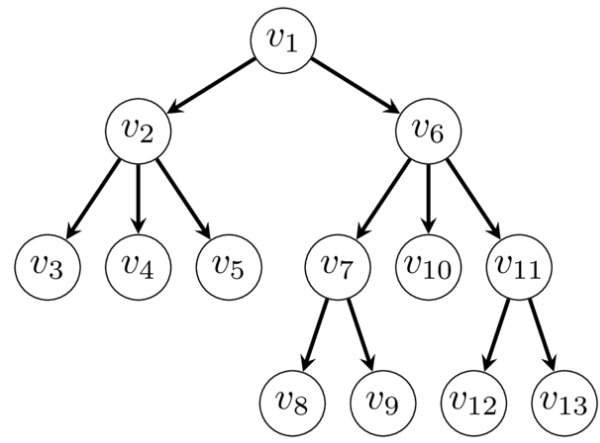

Fig. 1. A rooted tree $T_{v_{1}}$ with the root $v_{1}$. 
A vertex $u$ is said to be the parent of the vertex $v$, denoted by $p(v)$ if $(u, v) \in E\left(T_{r}\right)$ and in such a case, the vertex $v$ is said to be a child of the vertex $u$. The children of a vertex $v \in V\left(T_{r}\right)$ are the set of all vertices $W \subseteq V\left(T_{r}\right)$ such that $(v, w) \in E\left(T_{r}\right)$ for all $w \in W$. A vertex having no children is said to be a leaf vertex. For a vertex $v$ let $S T(v)$ be the subtree induced by all the vertices $w$ such that there is a path from $v$ to $w$ in $T_{r}$ [4].

An edge-coloring of a graph $G$ is an assignment of colors to the edges of the graph so that no two adjacent edges have the same color. An edge-coloring of a graph $G$ with the colors $1, \ldots, t$ is an interval t-coloring if all the colors are used, and the colors of edges incident to each vertex of $G$ form an interval of integers. A graph $G$ is interval colorable if it has an interval t-coloring for some positive integer $t$. The set of all interval colorable graphs is denoted by $\mathfrak{N}$. The concept of interval edge-coloring of graphs was introduced by Asratian and Kamalian [2] in 1987. This means that an interval t-coloring is a function $\alpha: E \rightarrow\{1, \ldots, t\}$ such that for each edge $e$ the color of that edge $\alpha(e)$ is an integer from 1 to $t$, for each color from 1 to $t$ there is an edge with that color and for each vertex $v$ all the edges incident to $v$ have different colors and the set of these colors forms an interval of integers. For an interval coloring $\alpha$ and a vertex $v$ the set of all the colors of the incident edges of $v$ is called the spectrum of that vertex in $\alpha$ and is denoted by $S_{\alpha}(v)$. The smallest and the largest numbers in $S_{\alpha}(v)$ are denoted by $\underline{S_{\alpha}}(v)$ and $\overline{S_{\alpha}}(v)$.

In [1] it was shown that every tree is from $\mathfrak{N}$. In this paper, we consider the case where there are strict restrictions on the spectrums and the problem is to find an interval t-coloring that meets those restrictions. In [6] a solution for the simplified version of this problem was provided when the restrictions are on the spectrums, the restrictions are strict, and all the spectrums contain the color 1. In [8] another limited version was considered where each vertex had at most one interval of forbidden colors. In [3] and [9] it was shown that for bipartite graphs with maximum degree equal to 3 and with strict restrictions on spectrums the problem of finding an interval t-coloring that meets the restrictions is an NP-complete problem. In [7] another problem with restrictions is considered for bipartite graphs where the restrictions are provided for one "part" of the bipartite graph.

\section{An $O(N)$ algorithm for edge coloring with given spectrums}

We will reduce the interval $t$-coloring of the tree with restrictions on spectrums to edgecoloring of a tree with restrictions on spectrums.

Problem 1: Given an arbitrary tree $T$ with $N=|V(G)|$ vertices and given strict spectrum restrictions $[l(v), r(v)]$ for every vertex $v$ with $1 \leq l(e) \leq r(e) \leq N$ and $r(v)-l(v)+1=d_{T}(v)$. Determine whether it's possible to have an interval $t$-coloring $\alpha: E(G) \rightarrow\{1, \ldots, N\}$ such that for each edge $e=(u, v), l(u) \leq \alpha(e) \leq r(u)$ and $l(v) \leq \alpha(e) \leq r(v)$.

Problem 2: Given an arbitrary tree $T$ with $N=|V(G)|$ vertices and given strict spectrum restrictions $S(v)$ for every vertex $v$ with $|S(v)|=d_{T}(v)$. Determine whether it's possible to have an edge coloring $\alpha$ such that for each edge $e=(u, v), \alpha(e) \in S(u)$ and $\alpha(e) \in S(v)$.

Note that in Problem 1 we require the restriction spectrums to be intervals while in Problem 2 the restriction spectrums can be arbitrary as long as the number of elements in the spectrum is equal to the degree of the vertex for all vertices. Problem 1 can be reduced to Problem 2 the following way. First if $\cup_{v \in V(G)}[l(v), r(v)] \neq[1, t]$ then it's impossible to have an interval $t$-coloring since the restrictions are strict. If the union forms an interval, then we just change the interval restrictions $[l(v), r(v)]$ for each vertex $v$ to a spectrum restriction $S(v)=\{l(v), l(v)+1, \ldots, r(v)\}$ and solve the second problem. If we find such an edge-coloring $\alpha$ then that coloring will also satisfy the first problem, because for every vertex the set of colors of its incident edges will be the $[l(v), r(v)]$ interval.

Now we will provide and $O(N)$ algorithm for the second problem. Without loss of generality, we can assume that $S(v) \subseteq\{1, \ldots, N\}$ and can assume $N \geq 2$ since the case $N=1$ is obvious. In that case every tree with $N \geq 2$ has a vertex $v$ with $d_{T}(v)=1$ (a leaf vertex). Let $v_{0}$ be an arbitrary leaf vertex. We are interested in the rooted tree $T_{v_{0}}$. Let the vertex connected to $v_{0}$ be $v_{1}$. In that case $T_{v_{0}}$ would look like the tree shown in Fig. 2.

We are going to solve the problem using dynamic programming on trees. For every vertex $v$ that has parent $p(v)$ let's denote the edge $(p(v), v)$ by $e_{v}$. If we can detect the color of $e_{v}$ for all the non-root vertices then we will detect the color of all the edges of the tree. Now imagine that for some vertex $v$ we already calculated the values for its children $u_{1}, \cdots, u_{k}\left(k=d_{T}(v)-1\right)$ and we have all the colors $c_{i}=e_{u_{i}}$ for every $1 \leq i \leq k$. In that case $\left\{c_{1}, \ldots, c_{k}\right\} \subset S(v)$ and all those colors should be different, otherwise it would be impossible to continue the coloring since the edge $e_{u_{i}}$ is also incident to vertex $v$ hence its color should be inside $S(v)$. But $\left|S(v) \backslash\left\{c_{1}, \ldots, c_{k}\right\}\right|=1$ this means there is a 
unique color $c \in S(v)$ that is not equal to any of the colors $c_{1}, \ldots, c_{k}$ hence $\alpha\left(e_{v}\right)$ should be the color $c$. Fig. 3 illustrates that.

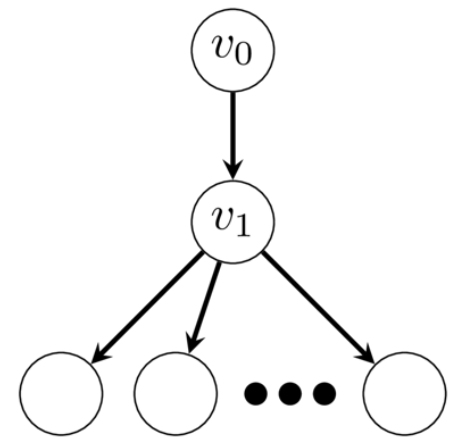

Fig. 2. The rooted tree $T_{v_{0}}$.

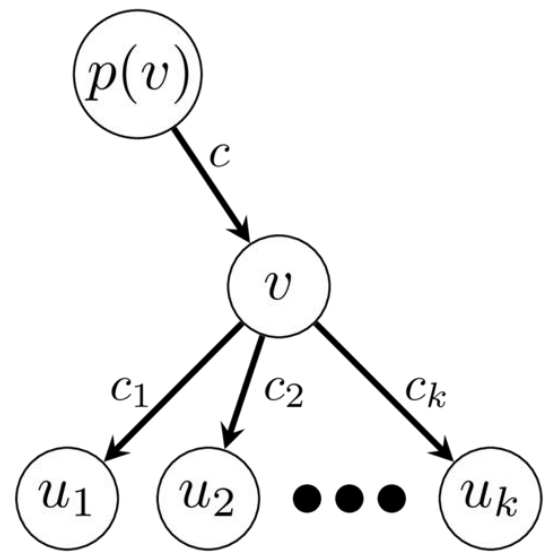

Fig 3. The colors of the children of vertex $v$.

This means that we can uniquely identify the color of edges incident to leaf vertices and construct all the other colors by moving up and subtracting all the used colors. If for some vertex we already constructed the colors of its children and there is conflict with the restrictions for the vertex $v$ we can continue constructing the color for the edge $e_{v}$ otherwise there is no coloring that satisfies the restrictions. Since for each vertex $v$ we would do $d_{T}(v)$ operations for finding the color that is different from the colors of the edges that connect to its children the complexity of the algorithm will be $\sum_{v \in V(T)} d_{T}(v)=2 \cdot|E(T)| \leq 2 \cdot N$ hence the algorithm is $O(N)$.

Conclusions. In this article we provided an efficient algorithm for checking and constructing interval coloring for trees on given strict spectrum restrictions. It uses dynamic programming.

\section{REFERENCES}

1. R.R. Kamalian "Interval colorings of complete bipartite graphs and trees", Preprint of the Computing Centre of the Academy of Sciences of Armenia, Yerevan, 1989

2. A.S. Asratian, R.R. Kamalian, Interval colorings of edges of a multigraph, Appl. Math. 5 (1987) $25-34$. (in Russian).

3. R.R. Kamalian. "Interval edge-colorings of graphs.” Doctoral Thesis, Novosibirsk, 1990.

4. D.B. West, Introduction to Graph Theory, Prentice-Hall, New Jersey, 1996.

5. Kuhn, H. (1955). The Hungarian method for the assignment problem. Naval research logistics quarterly, 2, 83-97. doi: 10.1002/nav.3800020109

6. Caro, Yair \& Schönheim, J. (1981). Generalized 1-factorization of trees. Discrete Mathematics. 33. 319321. 10.1016/0012-365X(81)90275-2.

7. A. S. ASRATIAN, "Investigation of Some Mathematical Model of Scheduling Theory," Doctoral dissertation, Moscow University, 1980

8. Kubale, M. (1989). Interval vertex-coloring of a graph with forbidden colors. Discret. Math., 74, 125-136.

9. Even, S., Itai, A. \& Shamir, A. (1976). On the Complexity of Timetable and Multicommodity Flow Problems. SIAM J. Comput., 5, 691-703. 